Advanced Computing: An International Journal ( ACIJ ), Vol.3, No.4, July 2012

\title{
IMAGE CONTENT IN LOCATION-BASED SHOPPING RECOMMENDER SYSTEMS FOR MOBILE USERS
}

\author{
Tranos Zuva ${ }^{1}$, Oludayo O. Olugbara ${ }^{2}$, Sunday O. Ojo ${ }^{3}$ and Seleman M. Ngwira ${ }^{4}$ \\ ${ }^{1,4}$ Department of Computer Engineering, Tshwane University of Technology, Pretoria, \\ South Africa, \\ zuvat@tut.ac.za \\ ${ }^{2}$ Department of Information Technology, Durban University of Technology, Durban, \\ South Africa \\ ${ }^{3}$ Faculty of Information and Communication Technology, Tshwane University of Technology, \\ Pretoria, South Africa
}

\begin{abstract}
This paper shows how image content can be used to realize a shopping recommender system for intuitively supporting mobile users in decision making. A mobile user equipped with a camera enabled smart phone combined with Global Positioning System (GPS) capabilities would benefit in using a recommender system for mobile users. This recommender system is queried by image sent by a smart phone together with the smart phone's GPS coordinates then the system returns a recommended retail shop together with its GPS coordinates, the image similar to the query image and other items on special offer. This recommender system shows a drastic reduction if not elimination of usage of text by mobile users using mobile devices when accessing the system. This paper presents the proposed recommender system and the simulated results of the recommender system. In summary the main contribution of this paper is to show how image retrieval, image content and camera enabled smart mobile device with GPS capabilities can be used to realize a location-based shopping recommender system for mobile users.
\end{abstract}

\section{KEYWORDS}

Recommender systems, image content, GPS, smart phone, e-commerce, image retrieval

\section{INTRODUCTION}

In the Information and Communication Technology (ICT) market there are several mobile technologies such as mobile data networks (General Packet Radio Service (GPRS) and Universal Mobile Telecommunications System (UMTS)), Global Positioning Systems (GPS), mobile phones and Personal Digital Assistants (PDAs) that are being used to offer online services to the global community whenever and wherever they are located [1]. These types of services are best suited to mobile users in places that they have never been to before and the user has to make a choice from a number of available options. These types of people would want to know their way to certain places or where they are, where to get an item(s) and /or an activity of their choice. At cheaper cost recommender systems can be of great help to this group of people in order to find places or where they are and/or item(s) of their taste in places where

DOI : 10.5121/acij.2012.3401 
Advanced Computing: An International Journal ( ACIJ ), Vol.3, No.4, July 2012

there are so many options to choose from [2]. These users would want to satisfy their short term needs so it is imperative that when they search for information, the output must be precisely accurate instantly or after a short time of feedback. The user must be satisfied with output from such applications. Recommender systems belong to a class of personalized information filtering technologies that aim to meaningfully suggest which items or products available might be of interest to a particular user [3-4]. Recommender systems have been applied in many diverse areas including e-commerce, advertisement, news, document management and e-learning [5]. They are one of the most popular tools provided in e-commerce to accommodate customer shopping needs with merchant offers [6]. These systems make recommendations using three fundamental steps: acquiring preferences from the user's input data, computing recommendations using proper methods and presenting the recommendation to the user [5]. All existing recommendation techniques can be classified into four fundamental categories that is Collaborative Filtering (CF), Information Filtering (Content-Based Filtering (CBF)), Knowledge-Based filtering (KBF) and Hybrid Filtering (HF). For in depth information about these techniques refer to [7-11].

The goal of the recommendation process is to efficiently find a set of items that match user desired item. The immediate motivation for the study is the following mobile recommendation problem.

Suppose Clarion leaves home with a location and a camera enabled mobile device for shopping and on getting to a nearby shop, she is faced with the option of window shopping. Moreover, assume Clarion finds it difficult locating a shop to find her choice of item. Instead of Clarion asking for help from someone, who probably has no adequate information, it would be prudent for her to rely on a kind of a mobile shopping recommender system to generate item-shop pair recommendations. Consequently, the problem is, with the aid of a mobile device carried by Clarion, how can she be helped to locate the nearest alternative shop to find an item to procure at affordable price and also to access relevant information on the item?

The rich contents of images and possible annotation of images with textual information make image retrieval technique [12] a useful technology for information search. The main idea of our approach is that with a camera and a location detector equipped mobile device, Clarion can point at an item in a retail or a wholesale outlet, take the image of the item (henceforth called logo), send the logo wirelessly to a remote server and receive useful information and recommendations. Among these mobile devices, cell phone is becoming a primary platform for information access for online mobile-users $[11,13]$. In this research a cell phone, smart phone and mobile device will be used interchangeably.

The goal of our approach is to (a) maximize the similarity between a recommendation and a query item, (b) minimize the location proximity between a user and a recommendation, (c) minimize the procurement price of a recommendation and (d) maximize the recommendation bait, which indicates some incentives that can influence one's preference for a recommendation.

The remainder of this paper is organized as follows:

Section II discuses the proposed recommender system and Section III the recommendation method employed is explained. In Section IV we give the simulated results of recommendation system and Section V we give a concluding remark and briefly discuss our future work. 


\section{Proposed ReCommender System}

The architecture of the proposed recommender system is shown in figure 1 . The client-side application running on a mobile device is responsible for acquiring query information and displaying personalized results for a mobile user. The primary input to the client application is a logo. It is also used for visualization of results based on the logo and the coordinates of the recommended retailer. Alternatively, the server-side application is equipped with image retrieval functionality and recommendations ranking technique to facilitate image based information search and recommendations generation. Sales item information is usually posted to a server through a web portal by registered sales retailers or wholesalers, thereby making the search results reliable. What makes our system interesting is the context of integrated wireless connectivity, location detection and digital imaging hardware. What makes it potentially useful is the assistance it offers to mobile users for discovering relevant business information and for decision support. Besides, retailers and wholesalers need not be online for their services to reach a large community of customers. Offline retailers and wholesalers would be able to advertise their sales services on the web using the resources provided by the recommender system. Then a large community of customers would be able to efficiently locate these services with the assistant of mobile devices running the recommender system client application.

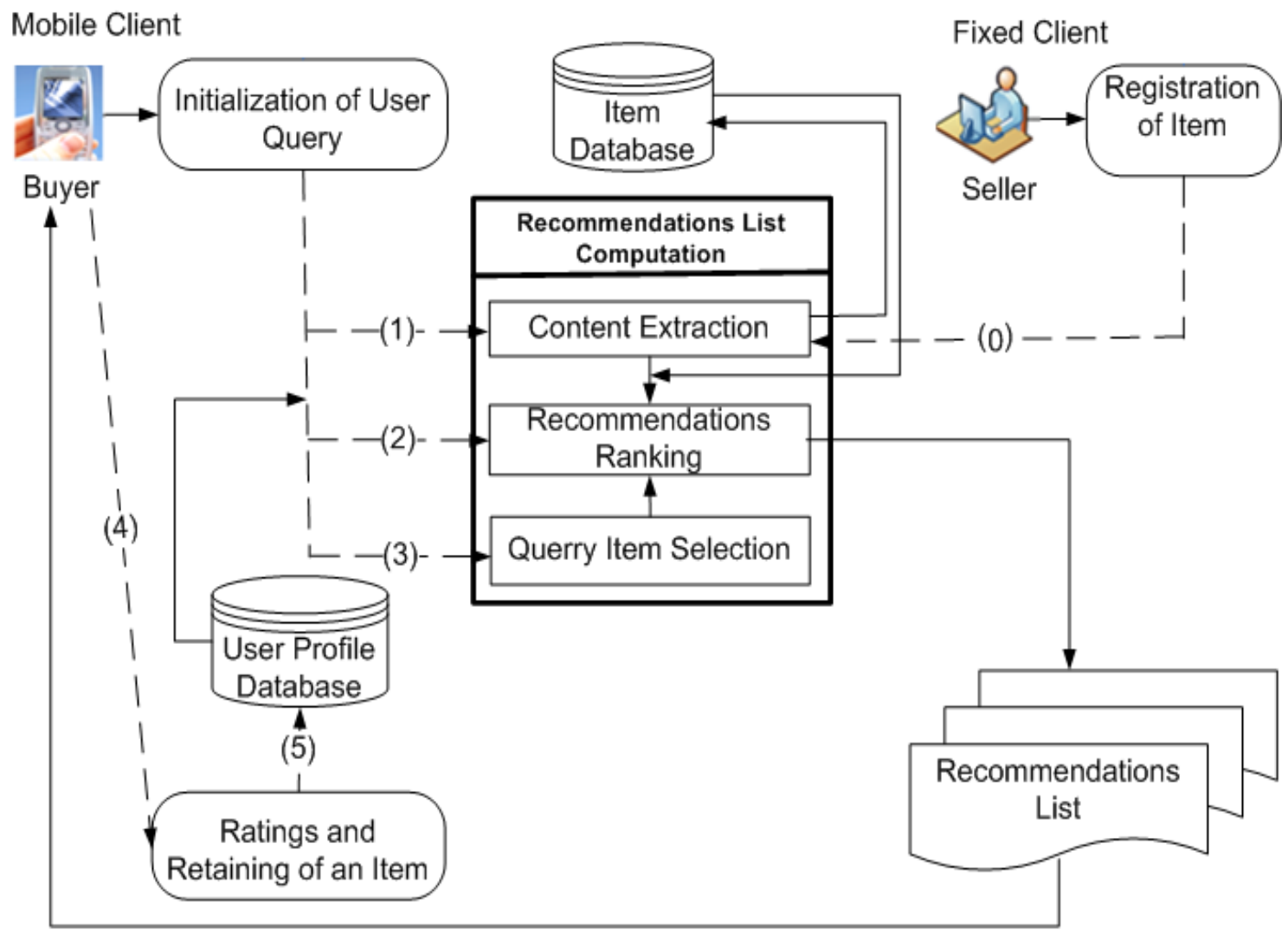

Figure 1. Architecture of the Recommender System

The shopping items stored in the database or used as queries are segmented using Active Contours Without Edges (ACwE) [14] and represented by Adaptive Kernel Density Feature Points Estimator (AKDFPE) [15]. The cosine similarity algorithm is used to match the query 
Advanced Computing: An International Journal ( ACIJ ), Vol.3, No.4, July 2012

image and the images in the database [16-17]. The recommender system was coded using Matlab programming language.

\section{RECOMMENDATION METHOD}

This section provides detail information about item and user profile representations modelling, recommendation process and user interaction modelling and recommendations list computation process of a shopping recommender system for mobile users.

The goal of our item representation is to model features that are common to large classes of items. In reality, this makes the system suitable as an e-Commerce recommender that can be used for sales of many different item types. We achieve this goal by capturing essential item information such as unique identifier, name, class, logo, price, payment method, shop and location. Specific features that are unique to each item are also captured and stored in the item database. Items in the database are identified by their logos (L). As a result, the recommendation method compactly represents items information as a feature vector of $m$ values as

$$
i=\left(i_{1}, i_{2}, i_{3}, \ldots \ldots . . i_{m}\right)
$$

where $i_{i}$ may be numeric, nominal or set of numbers.

A typical example of an item feature vector is:

$i=\left(L_{i d}, G P S s, L_{G P S s}\right)$ where $L_{G P S s}=\left(L_{i d}\right.$, price - range, size - range, promotion,,..$\left.e t c\right)$

where $L_{i d}$ is identifier of the logo, GPSs is where the item can be found, $L_{G P S s}$ is the set of features of the item at different locations, price-range is the range of the item in its various sizes, size-range is the sizes available of the item and so forth.

The user profile is also modelled as a feature vector of $n$ values

$$
u=\left(u_{1}, u_{2}, u_{3}, \ldots \ldots . . u_{n}\right)
$$

where $u_{i}$ may be numeric, nominal or set of numbers.

A typical example of a user feature vector is:

$$
u=\left(G P S, I_{A F S S}\right) \text { where } I_{A F S S}=(\text { price }- \text { range }, \text { size }- \text { range }, . . e t c)
$$


Advanced Computing: An International Journal ( ACIJ ), Vol.3, No.4, July 2012

where GPS is where the user is located at the time of querying the system, $I_{A F S S}$ is the set of average features of the items bought by user previously that is price-range, size-range and so forth.

When the mobile client sends a logo together with GPS coordinates to the system the following steps are taken:

(1) Searches the logo in the database

(2) Finds the logo similar to the query logo

(3) Looks at the GPS coordinates of locations where the item can be found

(4) Calculates the distances between the mobile client and the retail locations

(5) Rank the locations according to distances calculated in 4

(6) Calculate the similarity between $I_{A F S s}$ and $L_{G P S s}$ (acceptable distances)

(7) The final ranking is done taking into consideration the 5 and 6

(8) Recommendation is send to the mobile client with GPS coordinates of the chosen location, promotions and special offers.

In this research the distance calculated in step 4 is done using the following formula [18] in (5):

$d_{u L}=2 * a \sin \left(\operatorname{sqrt}\left((\sin ((\text { lat } 1-\text { lat } 2) / 2))^{2}+\cos (\right.\right.$ lat 1$) * \cos ($ lat 2$) *(\sin ((\text { lon } 1-\text { lon } 2) / 2))^{2}$

where $\{$ lat 1, lon 1$\}$ and $\{$ lat 2, lon 2$\}$ are GPS coordinates for the mobile client and the retail location respectively. It is very important to note that lat and lon stand for latitude and longitude respectively. North latitudes and west longitudes are taken as positive and south latitudes and east longitudes are taken as negative.

In step 6 the similarity is calculated using the Cosine similarity formula in (6):

$$
\operatorname{sim}\left(I_{A F S S}, L_{G P S S}\right)=\frac{\sum I_{A F S} * L_{G P S}}{\sqrt{\sum I_{A F S}^{2}} \sqrt{\sum L_{G P S}^{2}}}
$$

In step 7 the similarity values for ranking are calculated using the following formula in (7):

$$
R(\operatorname{sim})=\operatorname{sim}\left(I_{A F S s}, L_{G P S s}\right) * \partial_{u L}
$$

where $\partial_{u L}$ is normalized $d_{u L}$ and then transformed to range $[0,1]$ and $\partial_{u L}$ is calculated in (8) as:

$$
\partial_{u L}=\frac{1}{2}\left(1-\frac{d_{u L}}{\sqrt{d_{u L}+a}}\right)
$$

In step 8 the biggest $R(\operatorname{sim})$ is the one that is recommended to the user. 


\section{Results}

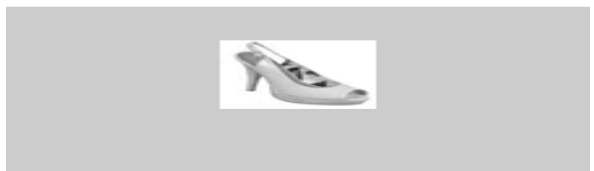

Figure 2. Query Image

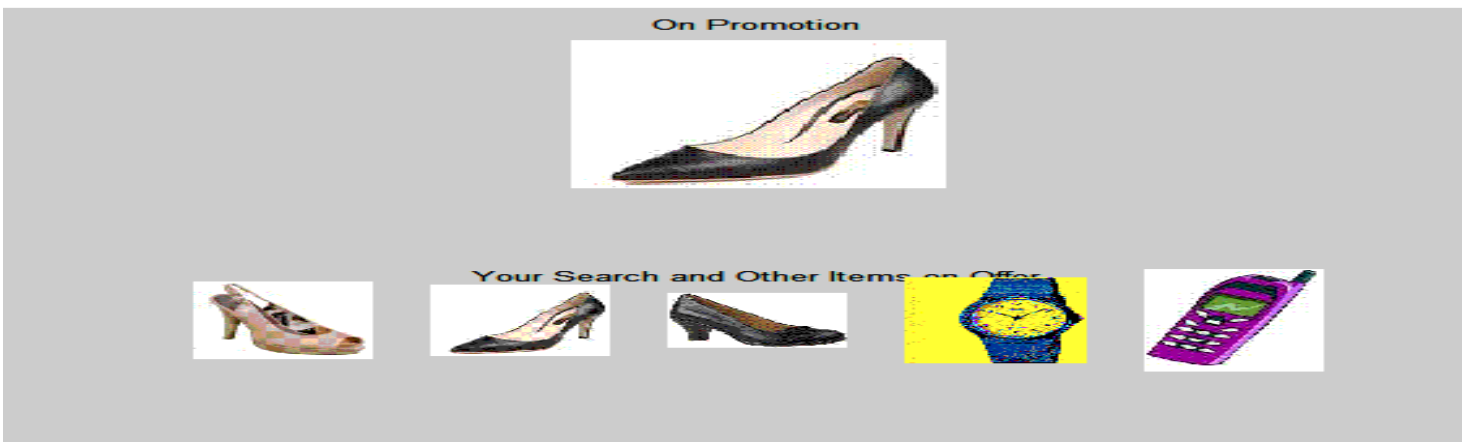

Figure 3. Results from the Shopping Recommender System

Figure 1 is an image captured at a retail shop and Figure 2 is the recommended results without GPS coordinates because the recommended retail shop is the one the user is located. The result shows the retrieved images and the other images of items on special offer in the retail shop.

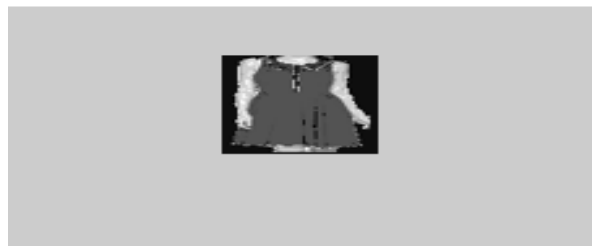

Figure 4. Query image captured by a camera enabled mobile device

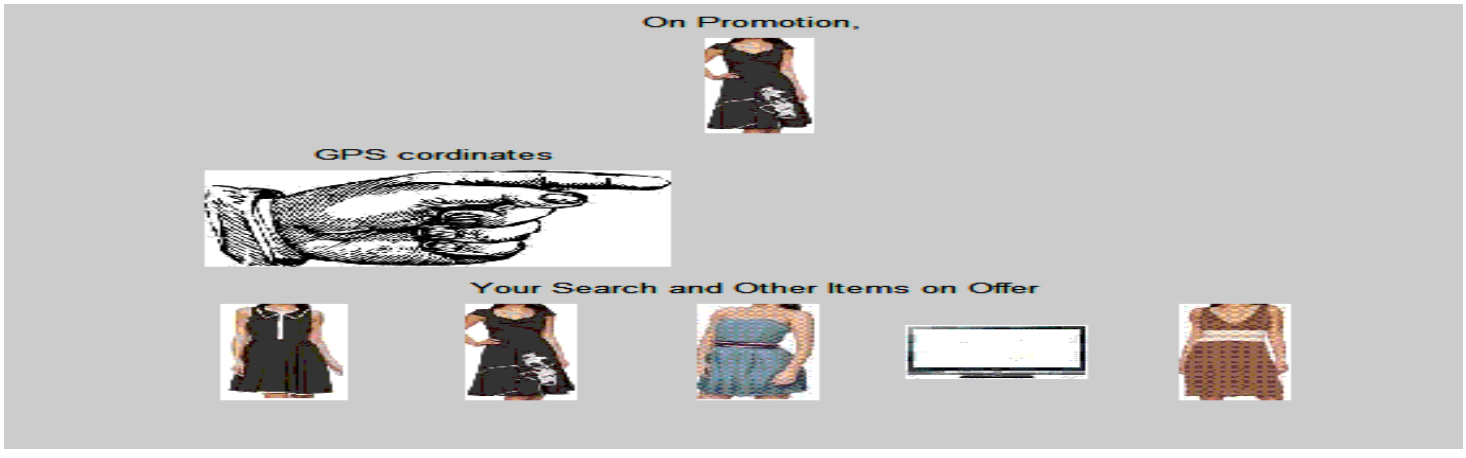

Figure 5. A result from the Shopping Recommender System with GPS coordinates for Retailer

Figure 4 shows the results from the query image in figure 3. The result comes with GPS coordinates of the retail shop where the mobile user can acquire the recommended item and also the items on special offer in that retail shop. 
Advanced Computing: An International Journal ( ACIJ ), Vol.3, No.4, July 2012

\section{Conclusion}

The recommender system gave a solution to the problem of creating a query by texting using a very small keypad of the mobile device. As they say "A picture is worth a thousand words" the image recommender system eliminate the spelling problem, keying text and ambiguity problems in query using text. The texting has been reduced drastically if not eliminated. The image retrieval operates at $100 \%$ precision when the recall is $10 \%$ meaning our recommender system operates at $100 \%$ image retrieval since the image retrieval of the recommender system is less than $10 \%$ recall. This has enabled the system to maximize the similarity between a recommendation and a query item. The system enables mobile users to get an item of their choice in the proximity of their location at their acceptable price. The mobile user also gets to know about items on special offer at the recommended retail shop. The retail shop owner is capable of selling goods without have to be online as a person since this system can be used by so many retail owners.

In future the system must be able to accept feedback from the user so that it can refine the recommendation. The system must also be able to allow the mobile user to add other factors to be considered in making recommendations such as weather but using icons. The system should be text-free to be able to have effective and efficient recommender system for mobile users.

\section{REFERENCES}

[1] D. Lu and Q. Weng, "A survey of image classification methods and techniques for improving classification performance," International Journal of remote Sensing, vol. 28, pp. 860-870, 2007.

[2] O. O. Olugbara, et al., "Exploiting Image Content in Location-Based Shopping Recommender Systems for Mobile Users," International Journal of Information Technology \& Decision Making, vol. 9, pp. 759-778, 2010.

[3] T. Bogers and A. v. d. Bosch, "Collaborative and Content-based Filtering for item Recommendation on Social Bookmarking Websites," in ACM RecSys '09 Workshop on Recommender Systems and the Social Web, New York, USA, 2009, pp. 9-16.

[4] A. Gunawardana and C. Meek, "A Unified Approach to Building Hybrid Recommender Systems," in Proceedings of the 2009 ACM Conference on Recommender Systems, New York, 2009, pp. 117-124.

[5] C.-L. Huang and W.-L. Huang, "Handling sequential pattern decay:Developing a two-stage collaborative recommender system," Electronic Commerce Research and Applications, vol. 8, pp. 117-129, 2009.

[6] W.-S. Yang, et al., "A location-aware recommender system for mobile shopping environments," Expert Systems with Applications, vol. 34, pp. 437-445, 2008.

[7] Z. Chen, et al., "A Collaborative Filtering Recommendation Algorithm Based on User Interest Change and Trust Evaluation," Internation Journal of Digital Content Technology and its Applications vol. 4, pp. 106-113, 2010.

[8] J. B. Schafer, et al., "Collaborative Filtering Recommender Systems," in The Adaptive web, Springer-Verlag, Ed., ed Berlin, Heidelberg, 2007, pp. 291-324.

[9] P. Melville and V. Sindhwani, "Recommender Systems," in Encyclopedia of Machine Learning, S. Verlag, Ed., ed Berlin: Springer, 2010, pp. 1-9.

[10] M. J. Pazzani and D. Billsus, "Content-based Recommendation Systems," in The Adaptive Web, methods and Strategies of Web Personalization, 2007, pp. 325-341. 
Advanced Computing: An International Journal ( ACIJ ), Vol.3, No.4, July 2012

[11] F. Ricci, "Mobile Recommender Systems," IT \& Tourism, vol. 12, pp. 205-231, 2010.

[12] Y. H. Ang, et al., "Image Retrieval Based on Multidimentional Feature Properties," in in Proc. SPIE: Storage Retrieval Image Video Databases III. 2420, San Diego/La Jolla, USA, 1995, pp. 47-57.

[13] M. Gabbouj, et al., "Content based Image Retrieval for Connected Mobile Devices," in Image Rochester, New York, 2005, pp. 255-264.

[14] T. F. Chan and L. A. Vese, "Active Contours Without Edges," IEEE, vol. 10, pp. 266-277, 2001.

[15] T. Zuva, et al., "Kernel Density Feature Points Estimator for Content-Based Image Retrieval," Signal \& Image Processing: An International Journal (SIPIJ), vol. 4, pp. 103-111, 2012.

[16] C.-C. Chen and H.-T. Chu, "Similarity Measurement Between Images," in Proceedings of the 29th Annual International Computer Software and Applications Conference, 2005.

[17] S.-H. Cha, "Comprehensive Survey on Distance/Similarity Measures between Probability Density Functions," International Journal of Mathematical Models and Methods in Applied Sciences, vol. 1, pp. 300-307, 2007.

[18] J. B. Adair and M. Turnbull, A procedure for calculating great circle distances between geographic locations: Council for Advanced transportation Studies, the University of Texas at Austin, 1974. 